Cahiers « Mondes anciens »

ANCIENS

Histoire et anthropologie des mondes anciens

3 | 2012

Femmes de paroles

\title{
Vigne mariée ou fleur coupée
}

La mise en scène d'une parole féminine dans le carmen 62 de Catulle

Marine Bretin-Chabrol

\section{(2) OpenEdition}

Journals

Édition électronique

URL : http://journals.openedition.org/mondesanciens/651

DOI : 10.4000/mondesanciens.651

ISSN : 2107-0199

Éditeur

UMR 8210 Anthropologie et Histoire des Mondes Antiques

Référence électronique

Marine Bretin-Chabrol, « Vigne mariée ou fleur coupée », Cahiers « Mondes anciens » [En ligne], 3 | 2012, mis en ligne le 23 mai 2012, consulté le 05 mai 2019. URL : http://journals.openedition.org/ mondesanciens/651; DOI : 10.4000/mondesanciens. 651

Ce document a été généré automatiquement le 5 mai 2019.

\section{(c) (i) $\Theta$}

Les Cahiers «Mondes Anciens » sont mis à disposition selon les termes de la licence Creative Commons Attribution - Pas d'Utilisation Commerciale - Pas de Modification 4.0 International. 


\title{
Vigne mariée ou fleur coupée
}

\author{
La mise en scène d'une parole féminine dans le carmen 62 de Catulle \\ Marine Bretin-Chabrol
}

Rares sont les paroles de matrones dans l'espace public romain, mais plus rares encore celles des jeunes filles, doublement invitées à la retenue par leur sexe et par leur âge ${ }^{1}$. Les vierges exemplaires dont Tite-Live rapporte les actes mémorables restent silencieuses: Virginia, uirgo adulta, convoitée par le décemvir Appius Claudius, est défendue par les cris de sa nourrice, les exclamations de la foule, les discours de son fiancé et de son père, mais ne prononce pas une parole de tout l'épisode qui la met en scène (Tite-Live, 3, 44-48). L'une des seules circonstances où l'on puisse entendre la voix des jeunes filles libres dans l'espace public de Rome est lorsqu'elles forment des chœurs lors de rituels officiels comme les Jeux Séculaires. Deux chœurs respectivement composés de vingt-sept filles et vingt-sept garçons chantent alors, à l'unisson, l'hymne offert à Apollon et à Diane. Le texte du Carmen saeculare est écrit pour eux par un professionnel, comme Horace le fit pour les Jeux célébrés en 17 av. J.-C. Ces pratiques romaines, qui se présentent comme la restauration, à l'aube du principat d'Auguste, de pratiques romaines plus anciennes, intègrent des éléments renvoyant au monde grec ${ }^{2}$.

2 Claude CALAme (1997, p. 262-263) a montré comment, en Grèce ancienne, les chœurs de jeunes filles constituaient un lieu d'initiation permettant leur intégration à la communauté. À côté des performances exécutées lors des grandes cérémonies religieuses engageant la cité, les jeunes filles grecques chantent également lors de fêtes familiales, telles que les mariages (CALAme 1997, p. 83-85). Dans tous les cas, selon Claude calame (1997, p. 234), les chœurs de jeunes filles ont pour fonction de les préparer au rôle qu'elles auront à jouer dans la cité à l'âge adulte, à savoir celui d'épouses et de mères. Le genre poétique de l'épithalame trouve son origine dans ces pratiques chorales.

3 Pourtant composé dans un contexte culturel et énonciatif différent, puisque la performance dont il prétend garder la trace n'est pas réelle, le carmen 62, l'un des deux épithalames du recueil poétique de Catulle (avec le carmen 61), se présente comme la transcription d'un chant exécuté par deux chœurs alternés, l'un de filles, l'autre de garçons. Le poème, composé entre 62 et 54 av. J.-C. (date de la mort de Catulle), compte aujourd'hui 66 hexamètres dactyliques, auxquels devaient s'ajouter cinq vers 
supplémentaires dans la version originelle, entre les vers 32 et 33, et un refrain entre les vers 58 et $59^{3}$. Jeunes filles et jeunes gens développent dans ce texte un discours opposé sur le mariage : les garçons invitent les filles à accepter l'union, tandis que les jeunes filles s'y refusent. Deux métaphores végétales, celle de la fleur dans un jardin et celle de la vigne mariée, sont utilisées par chacun des deux groupes pour définir la condition féminine idéale. Du fait de cet affrontement, le sexe des exécutants est un trait signifiant du dispositif énonciatif. Le poème fait en effet entendre deux points de vue, l'un présenté comme masculin, l'autre comme féminin.

Il faut préciser cependant les catégories qui sont ici mises en scène par le poète, car le sexe n'est pas le seul paramètre en cause. Les garçons se désignent eux-mêmes comme iuuenes (v. 1) ou aequales (v.11), ce qui correspond à un groupe dont l'homogénéité est assurée à la fois par une parité de sexe, d'âge, et, en ce qui concerne la première de ces deux expressions, de statut social : les iunenes sont en effet des jeunes gens libres, en âge de porter les armes (NÉRAUDAU 1979, p. 98-101 et p. 238-239). Face à eux, les jeunes filles s'appellent elles-mêmes innuptae (v. 6) ou aequales (v. 32). Nous retrouvons l'idée d'une parité d'âge, mais le groupe est principalement défini par la situation de ces jeunes filles à l'égard du mariage. Si elles sont innuptae, "non mariées », c'est qu'elles sont dans la condition de l'être : ce sont donc des jeunes filles libres et nubiles ${ }^{4}$. Les deux groupes ne sont pas définis de façon strictement symétrique.

5 Par ailleurs, les deux discours sont la création d'un seul et même poète qui intègre cette pièce à un recueil caractérisé par la variété des genres poétiques pratiqués (élégie amoureuse, épigramme, épyllion ${ }^{5}$, épithalame), des tons adoptés, des formes énonciatives présentées, et par conséquent par la variété des discours tenus sur la sexualité. Est-il raisonnable d'imaginer, comme l'ont fait quelques critiques $^{6}$, que Catulle donne ici, par la voix des garçons, son propre point de vue sur le mariage? Cette hypothèse nous parait simpliste, car elle ignore les spécificités thématiques impliquées par chacune des formes poétiques du recueil: l'épithalame célèbre par essence le couple conjugal, l'élégie généralement celui des amants inconstants. Dans le dialogue mis en scène par Catulle, il nous semble plutôt que le poète propose un équivalent poétique au rituel de la deductio in domum mariti, séquence caractéristique de la cérémonie romaine de mariage, sans pour autant chercher à faire l'apologie de l'institution. La parole des jeunes filles innuptae est alors construite par le poète comme le discours typique et attendu d'une classe d'âge.

\section{Une joute poétique fictive}

Les épithalames de Catulle, les carmina 61 et 62, relèvent d'une énonciation fictive. Les critiques s'accordent en effet à penser aujourd'hui que, même si les circonstances ayant occasionné leur composition ont pu être réelles, ces textes n'ont pas été chantés lors d'une cérémonie ${ }^{7}$. Il semble bien qu'à l'époque de Catulle, à Rome, le rituel du mariage ne comportait pas de chants ${ }^{8}$. Pourtant, par de nombreux signes énonciatifs, le carmen 62 se donne à lire comme le texte d'une performance ${ }^{9}$ exécutée par deux chœurs déclamant en alternance des vers amébées, en hexamètres dactyliques.

7 Par son refrain traditionnel invoquant le dieu Hymen (Hymen o Hymenaee, Hymen ades o Hymenaee!) et par son adresse à l'astre du soir Hespérus (v. 20, v. 26, v. 35), le poème se présente comme un hymne ${ }^{10}$, cadeau fait à un dieu pour qu'il favorise l'entreprise humaine à l'occasion de laquelle il est prononcé. La fiction de la performance est construite dans le texte par l'emploi des deux premières personnes du pluriel (Consurgite, 
v. 1 ; cernitis, v. 6 ; nobis, v. 11 et v. 32), qui posent un rapport d'interlocution au sein de chaque chœur (v. $1-19$; v. 32 ; v. 39-58) ${ }^{11}$, entre chaque chœur et le dieu (v. 20-31; v. 33-38), puis entre le chœur des jeunes gens ${ }^{12}$ et la fiancée (v. 59-66). Les interlocuteurs sont clairement identifiés par des termes d'adresse au vocatif (iuuenes, innuptae, aequalis, Hespere, uirgo). Cette fiction est entretenue par la référence au présent de l'énonciation, grâce à l'emploi de temps et de modes verbaux appropriés, parfois précisés par des adverbes de temps qui en soulignent l'aspect : impératif (consurgite, v. 1 ; nunc... conuertite, v. 17), présent de l'indicatif (uesper adest, v. 1 ; tandem... tollit, v. 2), futur performatif (iam dicetur hymenaeus, v. 4) ou parfait à valeur de passé immédiat (exiluere, v. 8). Le contexte évoqué est celui de la célébration d'un mariage, plus précisément du moment où, après le repas de noces, la fiancée quitte la maison de ses parents ${ }^{13}$ pour gagner celle de son mari, accompagnée par une procession. Les historiens du mariage romain appellent cette séquence du rituel deductio in domum mariti (TREGGIARI 1991, p. 166). Catulle en évoque les étapes de façon beaucoup plus détaillée dans l'autre épithalame du recueil, le carmen 61, v. 76-165.

8 L'alternance des couplets attribués respectivement aux garçons et aux filles se fait sur le principe de la joute poétique en vers amébées ${ }^{14}$. La rivalité poétique entre les deux chœurs est un thème explicite dans le poème, puisqu'il s'agit de remporter une victoire ( uincere, v. 9 ; uictoria, v. 16), une palme (palma, v. 11), à l'issue d'un affrontement dans lequel les membres des deux chœurs se tiennent debout face à face (consurgite contra, v. 6). Le concours a pour objet la performance poétique elle-même, qui est présentée comme improvisée au prix d'une grande concentration (v. 12-17). Conformément aux habitudes de la poésie amébée, les strophes improvisées doivent se répondre : dicere iam incipient, iam respondere decebit (v.18), «elles vont commencer à chanter, nous devrons leur répondre ». Et l'on observe en effet que parmi les huit couplets complets du poème, six sont construits sur ce principe ${ }^{15}$ : même nombre de vers, similitude des structures syntaxiques et du lexique, parenté thématique et jeu d'oppositions.

9 Il semble que le choix de donner à un épithalame une forme de joute soit une originalité de Catulle. Bien que l'on trouve mention, dans le Bouclier du Pseudo-Hésiode, de la présence de deux chœurs, l'un masculin, l'autre féminin, lors d'une cérémonie de mariage, ces deux chœurs ne sont pas présentés comme entonnant des chants alternés. La partie chantée est réservée aux garçons, accompagnés de la flûte, tandis que les jeunes filles dansent au son de la lyre (Pseudo-Hésiode, Scut. 273 sq.). L'un des fragments des épithalames de Sappho se présente sous une forme dialoguée (dialogue entre la jeune fille et sa virginité, Sappho fr. 109 Reinach), un autre rapporte la parole du père de la mariée (Sappho fr. 123 Reinach), mais rien ne permet de conclure à une énonciation distribuée entre deux chœurs (CALAME 1997, p. 84). Plus généralement, l'enquête menée par Claude CALAME dans les Chœurs de jeunes filles en Grèce ancienne lui permet d'affirmer qu'à l'exception de l'exemple donné dans le Bouclier ${ }^{16}$, tous les témoignages portant sur la composition des chœurs d'épithalame les décrivent exclusivement comme des chœurs féminins ${ }^{17}$. La joute entre garçons et filles ne constitue donc pas une structure traditionnelle de l'épithalame grec.

10 Catulle emprunte la forme de la joute aux Idylles de Théocrite qui fondent le genre de la bucolique. Des Idylles, Catulle conserve la structure amébée et le thème de la rivalité poétique, mais il se démarque de Théocrite en faisant lutter deux chœurs plutôt que deux poètes individuels et en rapportant cette lutte à une opposition entre filles et garçons. 
11 Bien qu'il s'inscrive fictivement dans la tradition de l'épithalame grec, le carmen 62 se démarque donc des pratiques traditionnelles lorsqu'il met en scène l'opposition de deux chœurs distingués par leur différence de sexe. La rivalité poétique se double par ailleurs d'une rivalité rhétorique : la discussion entre filles et garçons sur le fait de savoir s'il faut se marier ou non évoque certains exercices préparatoires qui seront pratiqués, un demisiècle après Catulle, dans les écoles de rhétorique ${ }^{18}$. Les jeunes filles refusent le mariage au nom de l'honneur qui est attaché à leur virginité, tandis que les jeunes gens le valorisent en vantant son rôle social. Deux images végétales sont mises au service de leur argumentation.

\section{Fille-fleur ou femme-vigne : la relecture de métaphores traditionnelles au service d'une représentation du mariage romain}

Les métaphores et les comparaisons qui assimilent l'homme à une plante sont traditionnelles dans la poésie grecque (АUBRIOT 2001, p. 51-62) et certaines sont devenues des lieux communs que Catulle reprend ici ou là dans son recueil. L'image de la fleur, flos, connote la beauté $(61,91-94)$, la jeunesse $(17,14)$, le charme qui rend désirable ou membre d'une élite $(63,64)$. Les images végétales sont plus précisément une caractéristique du genre de l'épithalame et nombreux sont les commentateurs qui ont relevé le lien entre les comparaisons employées par Catulle et celles que l'on rencontre dans la poésie de Sappho. Deux fragments, en particulier, rapprochés par les éditeurs en raison de leur similitude de structure, paraissent être la source de la double comparaison de Catulle ${ }^{19}$. Ne nous sont restés que les deux comparés végétaux : une jacinthe foulée au pied par des bergers et une pomme perchée au sommet d'un arbre, inaccessible.

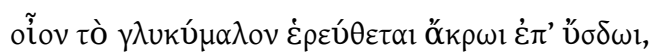

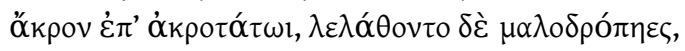

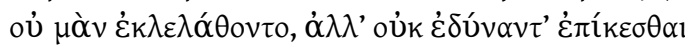

(Sappho, fr. 112 Reinach)

Comme on voit la pomme douce rougir au sommet d'une branche,

là haut sur la plus haute branche où les cueilleurs de pomme l'ont oubliée :

- non, ils ne l'ont pas oubliée, mais ils n'ont pas pu l'atteindre

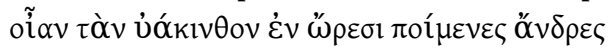

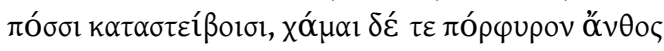

(Sappho, fr. 113 Reinach)

Telle que l'on voit, dans la montagne, une jacinthe foulée aux pieds

par des bergers, la fleur pourprée sur le sol < est tombée >

Cependant là encore, le poète donne une formulation nouvelle aux comparaisons dont il s'inspire: l'image du mariage des vignes, placée dans la bouche des garçons, est une image neuve. En s'opposant à la métaphore courante de la défloration, elle consacre la victoire des garçons dans la joute, mais elle permet aussi de donner une épaisseur nouvelle à l'image employée par les jeunes filles.

\section{Le jardin de l'innocence}

Les jeunes filles se comparent à une fleur protégée par les murs d'un jardin, admirée tant qu'elle reste intacte, mais fanée dès qu'on la cueille.

$V t$ flos in saeptis secretus nascitur hortis, ignotus pecori, nullo conuolsus aratro, quem mulcent aurae, firmat sol, educat imber; 
multi illum pueri, multae optauere puellae ;

idem cum tenui carptus defloruit ungui,

nulli illum pueri, nullae optauere puellae ;

sic uirgo, dum intacta manet, dum cara suis est ;

cum castum amisit polluto corpore florem,

nec pueris iocunda manet, nec cara puellis.

Hymen o Hymenaee, Hymen ades o Hymenaee!

(Catulle, 62, 39-48)

Comme une fleur, à l'abri dans l'enceinte d'un jardin, croît

ignorée du bétail, préservée des atteintes de la charrue ;

les brises la caressent, le soleil l'affermit, la pluie la nourrit ;

beaucoup de jeunes garçons, beaucoup de jeunes filles l'ont désirée ;

puis, lorsque, cueillie du bout de l'ongle, elle s'est fanée,

il n'y a plus de jeunes garçons ni de jeunes filles qui la désirent ;

ainsi, tant qu'une vierge reste intacte, elle est chère à tous les siens ;

quand une souillure a fait perdre à son corps la fleur de la chasteté,

elle n'est plus recherchée des jeunes garçons ni chérie des jeunes filles.

Hymen ô Hyménée, viens, Hymen ô Hyménée ! ${ }^{20}$

Le terme flos est employé à deux reprises dans ce couplet, une fois au sens propre, celui de " fleur », dans le cadre d'une comparaison (v. 39), une fois au sens figuré de "virginité » ( castum florem, v. 46). Il est intéressant que ce soit le terme générique qui soit employé plutôt que le nom d'une variété de fleur particulière, comme la jacinthe. La polysémie du mot latin révèle la banalité de l'image ${ }^{21}$. Le thème de la virginité de la fiancée est également un lieu commun de l'épithalame. Les jeunes filles reprennent ainsi deux éléments traditionnels de l'éloge de la fiancée : l'éloge de sa beauté qui la rend désirable, celui de sa chasteté qui la rend honorable ${ }^{22}$.

Mais en filant la comparaison avec l'univers végétal, le poète ravive les connotations liées au référent botanique. La fleur décrite ici possède un statut intermédiaire entre le monde sauvage et le monde cultivé. Bien que l'on ne sache rien des causes de sa naissance ( nascitur, v. 39), sa croissance n'est due qu'à l'action des éléments naturels que sont le vent, le soleil et la pluie (v. 41). Pourtant, elle pousse bien au sein d'un jardin, c'est-à-dire d'un espace créé et maîtrisé par une main humaine, qui a pris soin de l'entourer de murs (v. 39), et non dans les montagnes où Sappho décrivait la jacinthe.

17 L'analogie permet donc de définir la condition désirée par les jeunes filles du carmen 62 comme une condition sociale ${ }^{23}$. Bien que poussant à l'écart du bétail et de la charrue, qui peuvent la piétiner ou la couper, la fleur avec laquelle les jeunes filles se comparent n'est pas un être sauvage et isolé. Elle est admirée, convoitée (optauere) par des enfants des deux sexes, pueri et puellae (v. 42,44,47), qui appartiennent à la même classe d'âge qu'elle, puisque le terme puellam était déjà employé au v. 23 par les jeunes filles pour désigner la fiancée. De plus, la jeune vierge est aimée par les membres de sa famille proche (cara suis est, v. 45). On retrouve dans ce couplet une allusion au fait que le mariage constitue un arrachement de la jeune fille à sa mère, arrachement exprimé aux v. 21-22 au moyen du verbe auellere, qui peut également faire référence à une technique de reproduction des plantes consistant à prélever au pied d'un arbre un surgeon doté de racines ${ }^{24}$. Le mariage est vécu par les filles comme une transplantation en un sol étranger.

Les jeunes filles revendiquent leur double appartenance au foyer de leurs parents et à une classe d'âge, celle des pueri, groupe caractérisé par une certaine forme d'indétermination sexuelle, puisqu'il est mixte et vierge ${ }^{25}$, et ne connait de la sexualité que la séduction et le désir. Aux yeux des jeunes filles, le mariage constitue une infraction à cet ordre social 
dans lequel elles trouvent leur place. Il n'apporte que désordre et confusion. En mêlant les corps, il les souille (polluto corpore, v. 46), et ses effets ne sont perçus que comme la négation de l'état antérieur des jeunes filles, comme le révèle l'emploi de négations (v. $44 ; 47)$ et de termes dénotant la destruction ou la perte (defloruit, v. 43 ; amisit, v. 46). Les jeunes filles n'envisagent donc pas le mariage comme un changement de statut mais comme leur anéantissement en tant que jeunes filles : leur univers de référence est limité à leur classe d'âge.

\section{Le mariage des vignes}

Afin de persuader les jeunes filles de l'intérêt du mariage, les jeunes gens leur tendent un double miroir : la jeune fille vierge est comparée à une vigne « veuve » (uidua uitis), tandis que l'épouse est présentée comme une vigne « mariée à un orme » (ulmo conincta marito).

Vt uidua in nudo uitis quae nascitur aruo, numquam se extollit, numquam mitem educat uuam, sed tenerum prono deflectens pondere corpus iam iam contingit summum radice flagellum; hanc nulli agricolae, nulli accoluere iuuenci ; at si forte eadem est ulmo coniuncta marito, multi illam agricolae, multi coluere iuuenci; sic uirgo dum intacta manet, dum inculta senescit ; cum par conubium maturo tempore adepta est, cara uiro magis et minus est inuisa parenti.

< Hymen o Hymenaee, Hymen ades o Hymenaee! > (Catulle, 62, 49-59)

Comme une vigne veuve, qui pousse sur un terrain nu, ne peut jamais se déployer, jamais produire de doux raisins, mais, courbant son corps flexible sous le poids qui l'entraîne, fait bientôt toucher sa racine à l'extrémité de ses sarments ; il n'y a point de laboureurs, point de taureaux qui la cultivent; mais si on la marie à un ormeau où elle s'enlace, beaucoup de laboureurs, beaucoup de taureaux la cultivent ; ainsi, tant qu'une vierge reste pure, tant qu'elle vieillit privée de soins ; lorsque, mûre pour le mariage, elle a contracté une union assortie, elle est plus chère à son époux, moins à charge à son père. Hymen ô Hyménée, viens, Hymen ô Hyménée!

La métaphore de la vigne se trouve déjà employée par Sappho, dans un épithalame, pour louer la grâce d'un corps souple et flexible, qui est celui du fiancé (Sappho, fr. 117 Reinach). Mais Catulle modifie l'image en jouant d'abord sur l'inversion des genres. La vigne ne représente plus le mari mais l'épouse. C'est l'orme qui joue ici le rôle d'époux. Pour renforcer cette assimilation, le poète va jusqu'à jouer avec le genre grammatical du nom de l'arbre. Ulmus, comme tous les noms d'arbres de la $2^{\mathrm{e}}$ déclinaison est un nom féminin, mais Catulle choisit de substantiver l'adjectif maritus et d'en faire une apposition, pour pouvoir employer ce mot au masculin ${ }^{26}$. Il souligne ainsi la répartition des rôles dans le couple en fonction du sexe de chacun.

21 La comparaison employée par le chœur des garçons a suggéré aux critiques le rapprochement de ce passage avec le fragment dans lequel Sappho compare la fiancée à une pomme perchée au sommet de l'arbre. La pomme est un fruit, comme le raisin porté par la vigne. Mais Catulle déplace le point d'ancrage de la comparaison en faisant de l'épouse une vigne capable de porter des fruits et non le fruit lui-même. Alors que la poétesse grecque fait de la fiancée un objet désirable et inaccessible, qui se laissera peut- 
être conquérir par le fiancé le plus valeureux, Catulle utilise l'image pour caractériser le rôle de la femme en tant que mère.

Le poète romain se démarque encore de Sappho en inscrivant son image dans un univers de référence qui est celui des pratiques culturales de l'Italie antique. Le "mariage » des vignes est en effet une technique utilisée en Campanie et dans la plaine du Pô, qui consiste à utiliser le support d'un arbre vivant pour favoriser la croissance des sarments ${ }^{27}$. Pour décrire ce mode de conduite de la vigne, les agronomes romains emploient un vocabulaire qui a sans doute été emprunté par catachrèse à l'institution humaine du mariage (maritare, maritus), mais qui est devenu rapidement le vocabulaire technique propre à désigner cette pratique ${ }^{28}$. Le motif de la vigne mariée va devenir, dans la poésie romaine d'époque augustéenne, un signe caractéristique du paysage italien (Horace, Carm . 4, 5, 21-30 ; Epod. 2, 1-40 ; Ovide, Pont. 3, 8, 13-14). Dans les Géorgiques, Virgile fait référence à cette pratique pour opposer Lesbos et l'Italie :

Non eadem arboribus pendet uindemia nostris

quam Methymnaeo carpit de palmite Lesbos.

(Virgile, Georg. 2, 89-90)

Différente est la vendange qui pend à nos arbres

de celle que récolte Lesbos sur le sarment de Méthymne.

Dans l'Antiquité, l'île de Lesbos, où Sappho composait ses poèmes, était réputée pour ses vignes et son vin. Mais il est vraisemblable que, comme la plupart des vignes de Grèce et d'Asie, celles de cette île aient été cultivées sans support ${ }^{29}$. L'image du mariage des vignes est donc nécessairement une création romaine ${ }^{30}$.

L'union de la vigne et de l'orme, comme celle du lierre enserrant le tronc d'un arbre, traduit l'intimité du rapport amoureux et permet de caractériser le mariage comme le lieu du bonus amor ${ }^{31}$. Cependant ce ne sont pas les arguments de l'affection ou du plaisir qui sont mis en avant par les garçons dans cette strophe. De même que la conduite de la vigne sur le support d'un arbre est une technique mise au point par les hommes d'Italie pour améliorer les qualités naturelles de la plante, de même le mariage est décrit ici dans toute sa dimension sociale: le statut de l'épouse, tel qu'il est représenté ici, est proprement romain.

Dans le cinquième couplet, les garçons évoquaient déjà les aspects légaux du mariage. Les desponsa conubia, « les alliances promises » (v. 27), désignent les fiançailles, sponsio, accord passé entre le futur mari (ou son père) et le père de la mariée, deux hommes qui sont peut-être respectivement désignés au v. 28 au moyen des pluriels généralisants uiri et parentes (FORDYCE 1990, p. 257, ad. v. 27). Prolongeant ce discours, les garçons mentionnent explicitement, au vers 54 , le mari et le père de la mariée, faisant ainsi à nouveau référence à la dimension institutionnelle de l'union envisagée.

Dépourvue du support de l'orme, la vigne ne se dresse pas vers le ciel, mais retombe sur elle-même. On songe à la notion juridique d'infirmitas sexus, qui permet de justifier la mise sous tutelle des femmes. La faiblesse morale que l'on attribue à celles-ci est transposée en une faiblesse physique de la vigne, qui ne peut, seule, ni s'élever, ni fructifier. En revanche, l'union de la vigne et de l'orme vise à produire des fruits, comme le mariage romain a pour but de donner des enfants, liberos quaerere. Sans support, la vigne est décrite comme improductive, ce qui, bien entendu, n'a pas de fondement biologique. De même, la jeune fille célibataire est considérée comme stérile, sans que la chose ait davantage de fondement physiologique. La question est à nouveau d'ordre juridique : une fille non mariée ne peut inscrire ses enfants dans la lignée masculine de leur père. 

d'un mariage par capture, modalité liée dans certaines sociétés à la pratique de l'exogamie ${ }^{32}$. Après avoir peut-être été coiffée au moyen d'une lance (Ovide, Fast. 2, 560 ; Plutarque, Quaest. Rom. 87), la jeune fille est enlevée aux bras de sa mère pour être conduite dans la maison de son mari (Catulle, 61, 3 : rapis ; 56-59 ; Festus, p. 364 Lindsay). Elle verse alors des larmes rituelles (Catulle, 61, 85-86). Lorsqu'au terme de la procession, elle va passer le seuil de sa nouvelle demeure, elle est soulevée du sol et portée par les assistants du marié (Plutarque, Quaest. Rom. 29 ; Rom. 15, 5 ; TREGGIARI 1991, p. 163-168 ; BOËLS-JANSSEN 1993, p. 161-192; FAYER 2005, p. 512-562). Les anciens voyaient dans cette violence symbolique une référence au mythe fondateur du conubium romain, l'enlèvement des Sabines (Plutarque, Quaest. Rom. 29; 87; Rom. 15, 6; Festus, p. 364 Lindsay).

31 La résistance manifestée par les jeunes filles dans le carmen 62 se traduit dans des termes qui rappellent les étapes du rituel : l'arrachement au sein de la mère (v. 21-22), le motif de l'enlèvement (v. 32), la résistance de la fiancée (retinentem... natam, v. 22), les plaintes dénoncées comme feintes (donc peut-être rituelles) par les garçons (ficto... questu, v. 36) reprennent les étapes de la procession et le lien avec le mythe de l'enlèvement des Sabines est suggéré par l'image des ennemis s'emparant des vierges dans une ville prise (v. 24) $)^{33}$. À l'opposé, le discours des garçons, reprenant la substance du discours qu'aurait adressé Romulus aux jeunes filles enlevées, présente le mariage comme une résolution équitable du conflit: les jeunes filles ne seront pas réduites en esclavage, comme des captives, mais accèderont au respectable statut d'épouses et de mères (Tite-Live, 1, 9, 14-16). Catulle propose donc un équivalent poétique au rituel de la deductio in domum mariti. Le discours de résistance des jeunes filles est conçu comme la transposition de leur attitude rituelle, tandis que la victoire finale des garçons calque l'aboutissement nécessaire du mariage. Le débat recrée la dynamique qui se trouve à l'origine de la procession. 
indépendamment de l'affrontement avec les garçons. Elle ne représente pas un point de vue divergent sur le mariage, puisque la résistance qu'elle figure est au contraire une composante essentielle à la représentation mythique et rituelle du mariage romain.

\section{BIBLIOGRAPHIE}

ARKINS B. (1982), Sexuality in Catullus, Hildesheim-Zürich-New York.

ARKINS B. (1999), An Interpretation of the Poems of Catullus, Lewiston-Queenston-Lampeter.

AUBRIOT D. (2001), «L'homme-végétal : métamorphoses, symbole, métaphore », dans Delruelle E. et Pirenne-Delforge V. éd., Kêpoi. De la religion à la philosophie, Mélanges offerts à André Motte, Kernos Suppl. 11, Liège, p. 51-62.

BENVENISTE E. (1969), Le Vocabulaire des institutions européennes, 1. Economie, parenté, société, Paris. BOËLS-JANSSEN N. (1993), La Vie religieuse des matrones dans l'Antiquité, Rome.

CALAME C. (1996), L'Éros dans la Grèce antique, Paris.

CALAME C. (1997), Choruses of Young Women in Ancient Greece : Their Morphology, Religious Role and Social Functions, Lanham ; trad. remaniée de Calame C. (1977), Les choeurs de jeunes filles en Grèce archaïque I. Morphologie, fonction religieuse et sociale, Rome.

DELLA CORTE F. (1976), « Catullo, la vite e l'olmo », Maia 28, p. 75-81.

ELLIS R. (1889), A Commentary on Catullus ( $2^{\mathrm{e}}$ éd), Oxford.

ERNOUT A. et MEILLET A. (1985), Dictionnaire étymologique de la langue latine. Histoire des mots, $4^{\mathrm{e}}$ tirage revu par J. André, Paris.

FAYER C. (2005), La Familia Romana. Aspetti giuridici ed antiquari. Sponsalia, matrimonio, dote. Parte seconda, Rome.

FEDELI P. (1983), Catullus'Carmen 61, Amsterdam ; trad. de Fedeli P. (1972) : Il carme 61 di Catullo, Freiburg.

FORDYCE C. J. (1990), Catullus. A Commentary, Oxford.

Goud T. (1995), « Who speaks the final lines ? Catullus 62 : structure and ritual », Phoenix 49 (1), p. 23-32.

MCLENNAN J.-F. (1865), Primitive marriage. An Inquiry into the Origin of the Form of Capture in Marriage Ceremonies, Edimbourg.

MOREAU P. (1995), « Paroles des hommes, paroles des femmes », dans Dupont F. éd., Paroles romaines, Nancy, p. 53-63.

NĖRAUDAU J.-P. (1979), La Jeunesse dans la littérature et les institutions de la Rome républicaine, Paris. SCHEID J. (2005), Quand faire, c'est croire, Les rites sacrificiels des Romains, Paris. 
STIGERS E. S. (1977), « Retreat from the male. Catullus 62 and Sappho's erotic flowers », Ramus 6, p. 83-102.

THOMSON D. F. S. (1997), Catullus, Toronto.

TREGGIARI S. (1991), Roman Marriage. Iusti Coniuges from the Time of Cicero to the Time of Ulpian, Oxford.

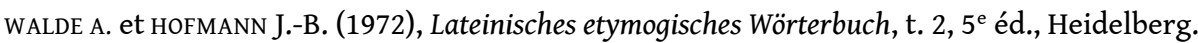

\section{NOTES}

1. Le roi Numa aurait édicté une règle de silence pour les femmes, selon Plutarque, Comp. Num. Lyc. 3, 10-11. Sur la parole des femmes dans l'espace public romain, cf. MOREAU 1995, p. 56-57.

2. Pour la description et l'étude de cette séquence rituelle, cf. SCHEID 2005, p. 97-110: «Les ambiguïtés du " rite grec" : l'exemple des Jeux Séculaires ".

3. Sur cette lacune, cf. les commentaires de ELLIS 1889, FORDYCE 1990, THOMSON 1997, ad. loc. et toute la discussion menée par GOUD 1995.

4. Catulle, 62, 32 : Hesperus e nobis, aequalis, abstulit unam, « Hespérus, ô mes compagnes, a enlevé l'une d'entre nous ». Les jeunes filles se définissent comme le groupe qu'est en train de quitter la fiancée. Cf. également Catulle, 61, 36-38: Vosque item simul, integrae uirgines, quibus aduenit par dies, agite in modum, dicite "O Hymenaee... », "Et vous aussi, toutes ensemble, chastes vierges, pour qui approche un jour semblable, allez, dites en mesure : "O Hyménée...” ».

5. L'épyllion est une très brève épopée. Dans le recueil de Catulle, ce genre est illustré par le carmen 64.

6. C'est ce que fait par exemple B. ARKINs (1982, p. 3, et 1999, p. 117), lorsqu'il s'interroge sur les attitudes contradictoires manifestées par Catulle dans le recueil à l'égard de la sexualité : comment le poète, amant d'une femme mariée, peut-il être le plus ardent défenseur de cette institution? Cependant, dans le détail de l'analyse, B. ARKINS est moins schématique.

7. L'unanimité est acquise pour le carmen 62 , mais la chose a été plus discutée pour le carmen 61. ELLIS 1889, p. 240 ; FEDELI 1983, p. 5 ; FORDYCE 1990, p. 235 et 254 ; THOMSON 1997, p. 348.

8. Philodème, De Musica (ed. Kemke) 68. 37-40 (vers 50 av. J.-C.) affirme que la pratique consistant à chanter des épithalames pendant les mariages est tombée en désuétude. Pour une description précise de la cérémonie romaine de mariage, cf. TREGGIARI 1991, p. 161-180 ; BOËLS-JANSSEN 1993, p. 161-192 ; FAYER 2005, p. 464-562. Cette cérémonie ne fait pas l'objet d'un rituel strict, mais peut adopter des formes variées. La procession qui conduit la fiancée à la maison de son époux peut être accompagnée de flûte, de cris, de jets de noix, de chants et de plaisanteries obscènes, mais aucune mention n'est faite par S. TREGGIARI de la présence de chœurs chantant un épithalame alors que les sources qu'elle utilise sont nombreuses (Térence, Ad. 903-910 ; Plaute, Cas. 798-810 ; Tite-Live, 1, 9, 12 ; Plutarque, Rom. 15, 2 sq. ; Quaest. Rom. 31 ; Martial, 1, 35, 6-7 ; 3, 93, 25 ; 12, 42, 4). Pour C. FAYER 2005, p. 517, la mention d'un hymenaeum que l'on chante et la citation du refrain Hymen, hymenaee, o hymen! dans les deux passages de Térence et de Plaute relevés par S. TREGGIARI suffiraient à prouver la pratique de l'épithalame dans la cérémonie romaine de mariage, mais plusieurs réserves doivent être apportées à cette suggestion : 1) Plaute et Térence ne sont pas contemporains de Catulle, et leur textes, inspirés de comédies grecques, ne constituent pas nécessairement le reflet fidèle de pratiques romaines. 2) Dans l'extrait de Casina, l'hymenaeum paraît se limiter au refrain lui-même, entonné à deux reprises par le futur mari, Olympion. 3) Seul l'extrait de Térence, dans lequel le futur mari, Eschine, déclare attendre la joueuse de flûte et «ceux qui doivent chanter l'hyménée (hymenaeum qui cantent)» (v.905), peut suggérer 
l'existence d'une pratique chorale de l'épithalame à Rome à l'époque de Térence. Nous n'avons donc aucune raison de remettre en cause l'assertion de Philodème citée au début de cette note.

9. Les paroles des deux chœurs ne sont pas rapportées par un narrateur qui relaterait les étapes de la cérémonie, contrairement à l'énonciation adoptée par Ausone dans le Cento nuptialis, v. 68-69: at chorus aequalis, pueri innuptaeque puellae, uersibus incomptis ludunt et carmina dicunt: «...».

10. Sur les différentes acceptions de ce terme, employé ici au sens de prière adressée à une divinité, cf. C. CALAME 1997, p. 74-76.

11. Pour T. GOUD 1995, p. 25, qui se distingue sur ce point de tous les éditeurs modernes, les trois premières strophes, qui introduisent la joute, ne seraient pas énoncées par l'ensemble du chœur, mais par le seul chef de chœur.

12. L'attribution de la dernière strophe, qui résout le conflit, est discutée : est-elle prononcée par les garçons qui remportent l'échange? par les filles qui acceptent ainsi leur défaite ? par les deux chœurs réunis? On y observe en effet la synthèse des thématiques affectives et juridiques respectivement développées par les filles et par les garçons dans les strophes précédentes. T. GOUD (1995, p. 32), appuyant sa démonstration sur une comparaison avec d'autres exemples de poèmes amébées grecs et romains (Théocrite, Id. 5 et 8 ; Catulle, 45, 62; Virgile, Ecl. $3 ; 7 ; 8$; Horace, Carm. 3, 9 ; Calpurnius Siculus, 2 et 4 ; Nemesianus, 2 et 4) et sur un rapprochement avec les formes de la cérémonie romaine de mariage, propose d'attribuer le dernier couplet au chorège du chœur de jeunes filles, qui jouerait ainsi le rôle de pronuba, matrone qui accompagne la fiancée tout au long de la cérémonie.

13. Catulle, 62,4 : iam ueniet uirgo, « maintenant va venir la vierge ».

14. Servius ad Virgile, Ecl. 3, 28 (cité par Goud 1995, n. 1 p. 23) : amoebaeum est quotiens quae canunt et aequali numero uersuum utuntur et ita se habet ipsa responsio, ut ad maius aut ad contrarium aliquod dicant, «Le poème est amébée lorsque les strophes chantées comportent le même nombre de vers et que la réponse elle-même est conçue de telle sorte que son énoncé soit une variation visant l'amplification ou l'antithèse » (traduction personnelle).

15. Ce sont les strophes 1 et 2,4 et 5, 8 et 9. Les strophes 6 et 7 sont incomplètes, mais il est probable qu'elles ont également été construites sur une structure symétrique.

16. Une autre exception est constituée par la scène de mariage représentée sur le bouclier d'Achille dans l'Iliade, où le chœur est constitué exclusivement de garçons, tandis que les jeunes filles sont spectatrices de la procession (Il. 18, 491 sq.).

17. Pindare, P. 3,16 sq. ; Théocrite, 18, 1 sq. ; mais aussi Eschyle fr. 43 Radt ; Prom. 555 sq. ; Euripide, Iphig. à Aulis 1054 sq.; Phae. 87 sq. et 217 sq. : références collectées par T. Goud 1997, p. 84.

18. Aelius Théon (rhéteur du début de la période impériale ou de la première moitié du deuxième siècle apr. J.-C., selon M. PATILlon, son éditeur dans la CUF, p. XVI) propose dans ses Progymnasmata comme exemple de «thèse ", la question : " Doit-on se marier ? " (Theon, 11, Peri theseos, 1. 3).

19. La comparaison entre le carmen 62 et ces deux fragments de Sappho est au cœur de l'étude de E.S. STIGERS (1977, p. 83-102).

20. Sauf mention contraire, toutes les traductions données ici sont celles de la CUF.

21. Cf. Catulle, 64, 402. Selon E.S. STIGERs (1977, p. 90-97), Catulle reprend bien ici à Sappho l'image de la jacinthe, mais il s'en démarque de façon polémique. Le discours des jeunes filles est discrédité par la simplification et la radicalisation de leur propos par rapport à celui que Sappho leur attribuait. Mais cette analyse repose sur le présupposé que le chœur des garçons est le porteparole de Catulle.

22. Cf. Catulle, 61, 86-93 (beauté), 19-20 (chasteté).

23. Ce point distingue encore le poème de Catulle des épithalames grecs dans lesquels la métaphore du mariage comme joug présente la jeune fille à marier sous l'aspect d'un animal 
sauvage à domestiquer. Cf. CALAME 1997, p. 238-244 et 1996, p. 165 : «Dans la pratique sociale, le rituel matrimonial a précisément pour fonction de faire de la contrainte sexuelle, symbolisée par la poursuite et le rapt, une domestication culturellement réglée et socialement admise : l'acceptation du pouvoir masculin, inscrite dans le rôle social (gender) de la femme adulte, s'exprime par la métaphore du passage à la civilisation ».

24. Pline utilise par exemple le nom auolsio, dérivé de ce verbe, dans la typologie des différentes techniques de reproduction des arbres qu'il adapte de Théophraste (Théophraste, Rech. plant. 2, 1, 1 ; Pline, Hist. nat. 17, 58 et 98).

25. Le chœur fictif chantant l'hymne à Diane du carmen 34 est ainsi composé de puellae et pueri integri, « jeunes filles et garçons chastes ».

26. Certains manuscrits présentent la leçon marita, mais nous suivons ici la lectio difficilior proposée par R. ELLIS 1889, p. 249, qui sert également de support à la réflexion de F. DELLA CORTE 1976, p. 75-81. La poésie de Catulle présente d'autres exemples de jeu sur le genre grammatical : pour souligner les effets de l'émasculation d'Attis, le poète fait passer les adjectifs qui se rapportent au personnage du masculin au féminin (Catulle, 63, 4 : stimulatus ; 8, citata).

27. Caton, Agr. 32 ; 47; Varron, Rust. 1, 8, 4 ; Virgile, Georg. 2, 358-361 ; 367-370 ; Columelle, Rust. 5, 6 ; Pline l'Ancien, Nat. 14, $10 ; 17,199-214$.

28. Caton, Agr. 32, 2. Pour ERNOUt et meILlet 1985, l'emprunt se serait fait dans l'autre sens : ce serait l'institution humaine qui aurait emprunté sa terminologie à la pratique culturale. Mais ce n'est pas l'opinion la plus couramment acceptée aujourd'hui, cf. WALDE et HOFMANN 1972, p. 41 ; HEY, s. u. maritus, -a, -um, TLL, t. 8, col. 403 ; BENVENISTE 1969, p. 246-247.

29. Pline mentionne le vin de Lesbos parmi les vins grecs réputés, mais ne donne pas d'indications sur le mode de conduite de ses vignes (Pline l'Ancien, Nat. 14, 73 ; 74 ; 97). Pour la culture de la vigne en Grèce et en Asie, cf. Varron, Rust. 1, 8, 1 et 5.

30. Le rhéteur grec Ménandre (vers 270 apr. J.-C.), traitant des épithalames, recommande de comparer le fiancé à un olivier ou à une pomme et d'exprimer l'intimité du rapport amoureux en recourant à l'image de l'union des arbres (Ménandre, Rhet. Gr. III 408, 14 sq. Spengel).

31. Cf. Catulle, 61, 34-35 ; 106-107 : dans ces deux passages, le verbe employé est implicare.

32. Ce type de mariage était pratiqué par les Doriens (Hérodote, VI, 65 ; Plutarque, Lyc. 15), mais aussi par de nombreux peuples du monde, selon MCLENNAN 1865, cité par R. ELLIS 1889, p. 210-211.

33. Pour une lecture de la cérémonie romaine de mariage comme mise en scène d'un rapt, dans laquelle la résistance de la jeune fille est l'un des éléments codifiés du rituel, cf. BOËLS-JANSSEN 1993, p. 162-163; FAYER 2005, p. 513.

\section{RÉSUMÉS}

Le carmen 62 de Catulle, épithalame fictif en vers amébées, met en scène l'affrontement d'un chœur de jeunes gens et d'un chœur de jeunes filles pour définir la condition féminine idéale (vierge ou mariée). Les comparaisons végétales, qui assimilent successivement la femme conquise à une fleur coupée et à une vigne mariée, y jouent un rôle argumentatif prépondérant. Dans cet affrontement, il ne s'agit pas pour le poète de présenter réellement le point de vue des femmes, mais de proposer, grâce à la dynamique de la joute, une traduction poétique au rituel romain qui donne à voir le mariage comme un enlèvement. 
Catullus'carmen 62 is a fictionnal epithalamion composed of amoebean verses. A young men chorus and a young women chorus argue about whether it is better for a woman to remain a virgin or to get married. Two comparisons with plants are opposed for the sake of argumentation. Girls compare married women to cut flowers, while boys identify them to vines supported by an elm. Catullus'goal is not to express the actual point of view of women, but to figure poetically - using the dynamic of the fight - the symbolic of the Roman wedding ceremony, which assimilates marriage to a capture.

\section{INDEX}

Keywords : Catullus, chorus of young women, comparison with plants, epithalamion, marriage, Rome

Mots-clés : Catulle, chœur de jeunes filles, comparaison botanique, épithalame, mariage, Rome

\section{AUTEUR}

\section{MARINE BRETIN-CHABROL}

Université Lyon III-Jean Moulin, HiSoMa (Histoire et Sources des Mondes antiques) - CEROR

(Centre d'Études et de Recherches sur l'Occident Romain) 